\title{
АЛГОРИТМЫ ВОССТАНОВЛЕНИЯ К-ОДНОРОДНЫХ ГИПЕРГРАФОВ ПО ВЕКТОРУ СТЕПЕНЕЙ СВОИХ ВЕРШИН
}

\section{ALGORITHMS FOR RESTORING K-HOMOGENEOUS HYPERGRAPHS BY THE VECTOR OF DEGREES OF THEIR VERTICES}

\author{
I. Beretskiy \\ I. Irbitskiy \\ E. Egorova \\ A. Mokryakov
}

Summary. This paper considers homogeneous hypergraphs (complexes) and methods for their reconstruction from vertex degree vectors. Two new algorithms for implementing a vector into a complex are considered: greedy (full iteration of simplices) and reductive (construction of simplices from vertices with the largest number of incident simplices remaining). A software package for restoring hypergraphs from vertex degree vectors using both of these algorithms is implemented.

Keywords: uniform hypergraph, the restoration of the hypergraph, the greedy algorithm, the reduction algorithm.

\section{Введение}

Д анная работа является продолжением идей, рассмотренных в работах Хакими С.П. [1], Миронова А.А. [2] и Мокрякова А.В. [3-5] о возможностях распределения вектора для $\mathrm{n}$-мерного случая. $\mathrm{B}$ их статьях был рассмотрен одномерный случай, а также представлены алгоритмы решения задачи, результат которой обобщает результаты, полученные для одномерного случая на двумерные.

Проблема реализации гиперграфов [6-7] ряда классов из вектора степеней его вершин обозначены при рассмотрении задач о распределении ресурсов, данных в виде векторов [8]. В работе рассматриваются четыре
Берецкий Игорь Сергеевич

Московский авиачионный институт (национальный исследовательский университет)

ISberetskij@mai.ru

Ирбитский Илья Сергеевич

Московский авиационный институт (начиональный исследовательский университет)

ILSirbitskij@mai.ru

Егорова Евгения Кирилловна

К.ф.-м.н., доцент, Московский авиационный институт (национальный исследовательский университет) egorovaek@mati.ru

Мокряков Алексей Викторович

К.ф.-м.н., доцент, Московский авиационный институт (национальный исследовательский университет); доцент, Российский государственный университет имени А. Н. Косыгина MokryakovAlVik@gmail.com

Аннотация. В работе рассматриваются однородные гиперграфы (комплек(ы) и методы их восстановления из векторов степеней вершин. Рассмотрены два новых алгоритма реализации вектора в комплекс: жадный (полный перебор симплексов) и редукционный (построение симплексов из вершин с самым большим из оставшихся количеством инцидентных симплексов). Реализован программный комплекс для восстановления гиперграфов из векторов степеней вершин, использующее оба указанных алгоритма.

Ключевые слова: однородный гиперграф, восстановление гиперграфа, жадный алгоритм, редукционный алгоритм.

класса гиперграфов, которые были определены в работах [9] при этом их определение было уточнено.

В своих трудах С.П. Хакими [1] поднимал проблему восстановления вектора в граф. В более поздних трудах [2-5, 8-10] идеи Хакими были расширены таким способом, что стало возможным получения всех возможных графов из исходного вектора, а не единственно из возможных. Однако при более комплексных случаях необходимо использовать понятие гиперграфа [11]. В работе [12] была предложено работать с экстремальными комплексами как с алгеброй, на которую распространены операции пересечения, дополнения и объединения. В работе [13] проведено исследование связи логических операций и вектора степеней вершин k-однородного ги- 
перграфа. Работы в данной области помогли применить экстремальные гиперграфы в области криптографии [14]. Данная работа также может применяться в этом направлении.

В работе приводятся алгоритмы восстановления вектора в комплекс на вид которого были наложены ограничения. Полученные алгоритмы могут использоваться в математической модели для получения качественного результата.

\section{1. К^ассы гиперграфов}

Рассмотрим алгоритмы восстановления гиперграфов некоторых классов из произвольных векторов. Подход был описан при рассмотрении проблем о распределении ресурсов, определенных в виде векторов. В процессе реализации комплексов выявляется проблема их большой вариативности. Восстановить комплекс из вектора степеней вершин становится возможным при условии, что на комплекс него будут наложены ограничения.

Введем обозначение $\Gamma(k, n)$ - гиперграф на $n$ вершинах с гиперрёбрами, которые могут содержать $k$ смежных вершин. Также обозначим $\Gamma^{1}(k, n)-$ гиперграф $\Gamma(k, n)$, у которого гиперрёбра не могут содержать повторяющиеся вершины. В противовес этому в гиперграфе $\Gamma^{\infty}(k, n)$ каждое гиперребро может содержать до $k$ одинаковых вершин.

Нижний индекс у $\Gamma(k, n)$ обозначает максимальный вес, который может быть у гиперребра: 1 - вес всех гиперребёр равен $1 ; \infty$ - вес каждого гиперребра должен быть положительным целочисленным значением.

Класс $\Gamma_{1}^{1}(k, n)$ соответствует -однородному гиперграфу, именно с ними мы в дальнейшем будем работать и для удобства будем писать сокращенно: $\Gamma_{1}^{1}=\Gamma_{1}^{1}(k, n)$.

\section{2. Известный алгоритм восстановления $\mathrm{K}$ - ОАНОРОАНОГО гиперграфа}

Для следующего алгоритма нам потребуется ввести обозначение: $l_{A}(0)-$ количество координат целочисленного неотрицательного вектора $A=\left(a_{i}\right), i=1, n$, равных нулю при $i \geq k$.

По работам $[5,6]$ известен следующий алгоритм:

\section{Алгоритм 1}

Пусть дан целочисленный вектор Б $=\left(a_{1}, \ldots a_{n}\right)$, где $n \geq k$ координаты которого больше или равны нулю.
Координаты вектора $Б$ упорядочены по невозрастанию. Чтобы получить гиперграф, будем по очереди отнимать от $k$ выбранных координат по единице. При каждом вычитании будем получать новое гиперребро. Таким образом постепенно будет построен гиперграф.

Шаг 1. Пусть вектор В $=\left(b_{1}, \ldots b_{n}\right)$, тогда $\mathrm{B}_{1}=\mathrm{B}-\mathrm{B}$, где

$$
\begin{aligned}
& b_{i}=\left\{a_{1}=\left\{a_{1}, \ldots, a_{k-1}, n-k+1-l_{\mathrm{B}}(0)\right\},\right. \\
& i=\underline{1, k-1} ; 1, i=\underline{k, n-k+1+a_{1}} .
\end{aligned}
$$

Шаг 2. Вектор $\mathrm{b}_{2}=\mathrm{b}_{1}-$ В, где

$$
\begin{aligned}
& b_{i}=\left\{a_{2}=\left\{a_{1}, \ldots, a_{k-2}, a_{k}, n-k-l_{\mathrm{B}}(0)\right\},\right. \\
& i=\underline{1, k-2}, i=k ; 1, i=\underline{k+1, n-k+a_{2}} . \\
& \text { Шаг } n-k . \mathrm{Б}_{n-k}=\mathrm{Б}_{n-k-1}-\text { В, где }
\end{aligned}
$$$$
b_{i}=\left\{a_{n-k}=\left\{a_{1}, \ldots, a_{k-2}, a_{n-1}, 1-l_{\mathrm{B}}(0)\right\}\right. \text {, }
$$$$
i=1, k-2, i=n-1 ; 1, i=n \text {. }
$$

Ш а г $n-k+1$. Если $a_{1}=0$, то сортируем вектор Б $\mathrm{b}_{n-k}$ , по невозрастанию и переходим к шагу 1 (при построении гиперребёр учитываем, что координаты перенумерованы), иначе находим вектор $\mathrm{b}_{n-k-1}=\mathrm{b}_{n-1}-\mathrm{B}$, где

$$
\begin{aligned}
& b_{i}=\left\{a_{n-k+1}=\left\{a_{1}, \ldots, a_{k-2}, a_{k}, n-k+1-l_{\mathrm{B}}(0)\right\},\right. \\
& i=\underline{1, k-2}, i=k ; 1, i=\underline{k+1, n-k+a_{n-k+1}} .
\end{aligned}
$$

В общей сложности из $a_{i}$ вершины можно вычесть до $C_{n}^{k}$. Алгоритмы завершает свою работу, когда все варианты вычитаний перебраны или некоторый вектор $\mathrm{b}_{p}=\overrightarrow{0}$.

П р и м е p 1. Построим гиперграф с гиперрёбрами по три вершины на основе вектора $\bar{~}=(10,7,6,4,3)$.

Построим ряд гиперребёр, содержащих по три вершины:

$$
\{1,2,3\},\{1,2,4\},\{1,2,5\},\{1,3,4\},\{1,3,5\},\{1,4,5\},\{2,3,4\} .
$$

В результате получаем семь наборов по три элемента в каждом и остаток из десяти элементов (четыре первых, три вторых и два первых).

\section{Окончательно имеем}

$$
\begin{aligned}
& \mathrm{E}=(10,7,6,4,3) ; \\
& \mathrm{b}_{1}=(9,6,5,4,3) ; \\
& \mathrm{b}_{2}=(8,5,5,3,3) ; \\
& \mathrm{b}_{3}=(7,4,5,3,2) ; \\
& \mathrm{G}_{4}=(6,4,4,2,2) ; \\
& \mathrm{b}_{5}=(5,4,3,2,1) ;
\end{aligned}
$$


$\mathrm{B}_{6}=(4,4,3,1,0)$;

$\mathrm{b}_{7}=(4,3,2,0,0)$;

Легко отследить количество исходных наборов элементов, доступных для разложения.

У данного алгоритма есть недостатки:

1. В случае неудачи он не даёт однозначного ответа на вопрос о возможности восстановления гиперграфа.

2. Его реализация недостаточно проста.

3. Скорость работы алгоритма также оставляет желать лучшего.

По этим причинам было решено разработать альтернативные алгоритмы восстановления гиперграфов класca $\Gamma_{1}^{1}$.

\section{3. Ж Канный алгоритм восстановления \\ к - олнородного гиперграфа}

Жадный алгоритм составляет симплекс из вершин, имеющих наибольшие степени, после чего проверяется, есть ли уже в гиперграфе такой симплекс. Если нет, он добавляется в гиперграф; при наличии такого симплекса, ищется возможность составить симплекс, которого в данный момент в гиперграфе ещё нет. В общем случае алгоритм работает (если гиперграф возможно восстановить из вектора, то он гарантированно будет восстановлен), однако, имеет высокую сложность и, как следствие, большое время работы на больших вектоpax.

\section{Алгоритм 2}

Шаг 0. Если vec $\not \equiv 0(\mid \bmod n)$, то вектор невосстановим в комплекс.

Шаг 1. Сортировка vес по невозрастанию.

Шаг 2. Если вектор состоит из нулей, то выйти.

Шаг 3. Составить из вершин вектора $(1, \ldots, n)$ симплекс. Если симплекс отсутствует в комплексе, добавить его в комплекс, уменьшить степени использованных вершин на 1 и перейти к шагу 1. Если такой симплекс уже есть, удалить последнюю добавленную вершину и взять следующую, с последующим переходом к шагу 3. Если составить симплекс невозможно, удалить последний добавленный симплекс и попытаться составить новый, взяв следующую вершину вместо последней добавленной.

Плюсом алгоритма является гарантия восстановления в n-комплекс, если для входного вектора это воз- можно. Минусом, очевидно, медленная скорость и, как следствие, большое время работы алгоритма.

\section{4. Фиксируюший алгоритм восстановления $\mathrm{k}$ - \\ о $А$ норо $А$ ного гиперграфа}

Фиксирующий алгоритм работает следующим образом: сначала вектор степеней вершин сортируется по невозрастанию. Далее, для -однородного гиперграфа фиксируются $(k-1)$ вершин со старшими степенями, а все оставшиеся вершины используются для составления симплексов, добавляемых в гиперграф. При обнулении старшей вершины происходит сортировка вектора, и процесс продолжается до тех пор, пока вектор не обнулится.

\section{Алгоритм 3}

Шаг 0. Если $v e c \not \equiv 0(\bmod n)$, то вектор невосстановим в комплекс.

Шаг 1. Сортировка vеc по невозрастанию.

Шаг 2. Если вектор состоит из нулей, то выйти.

Шаг 3. Фиксация (n-1) старших вершин вектора и составление гиперрёбер со всеми оставшимися вершинами. Если во время составления гиперребра одна из зафиксированных вершин обнулится, остановиться и перейти к шагу 1.

Алгоритм работает для случаев обычного графа и большинства случаев для 3-однородного гиперграфа (каждое гиперебро объединяет 3 вершины). На данный момент найдены контрпримеры векторов для общего случая, гиперграф из которых восстановим, но алгоритм не справляется с восстановлением. Поиск улучшений для работы алгоритма в общем случае продолжается.

\section{5. Программный комплекс}

Для реализации алгоритмов был использован язык С\# [23]. Этот язык является простым в освоении и относится к большому семейству языков с С подобным синтаксисом, который хорошо знаком программистам на языках C, C++, Java и JavaScript. Основными достоинствами С\# можно назвать возможность его использования с другими языками (Visual Basic, F\#) программирования в рамках одного приложения, типобезопасность, сборщик мусора, структурированная обработка исключений.

Для реализации клиентского приложения была выбрана технология для построения Desktop-приложений Windows Presentation Foundation (WPF). 


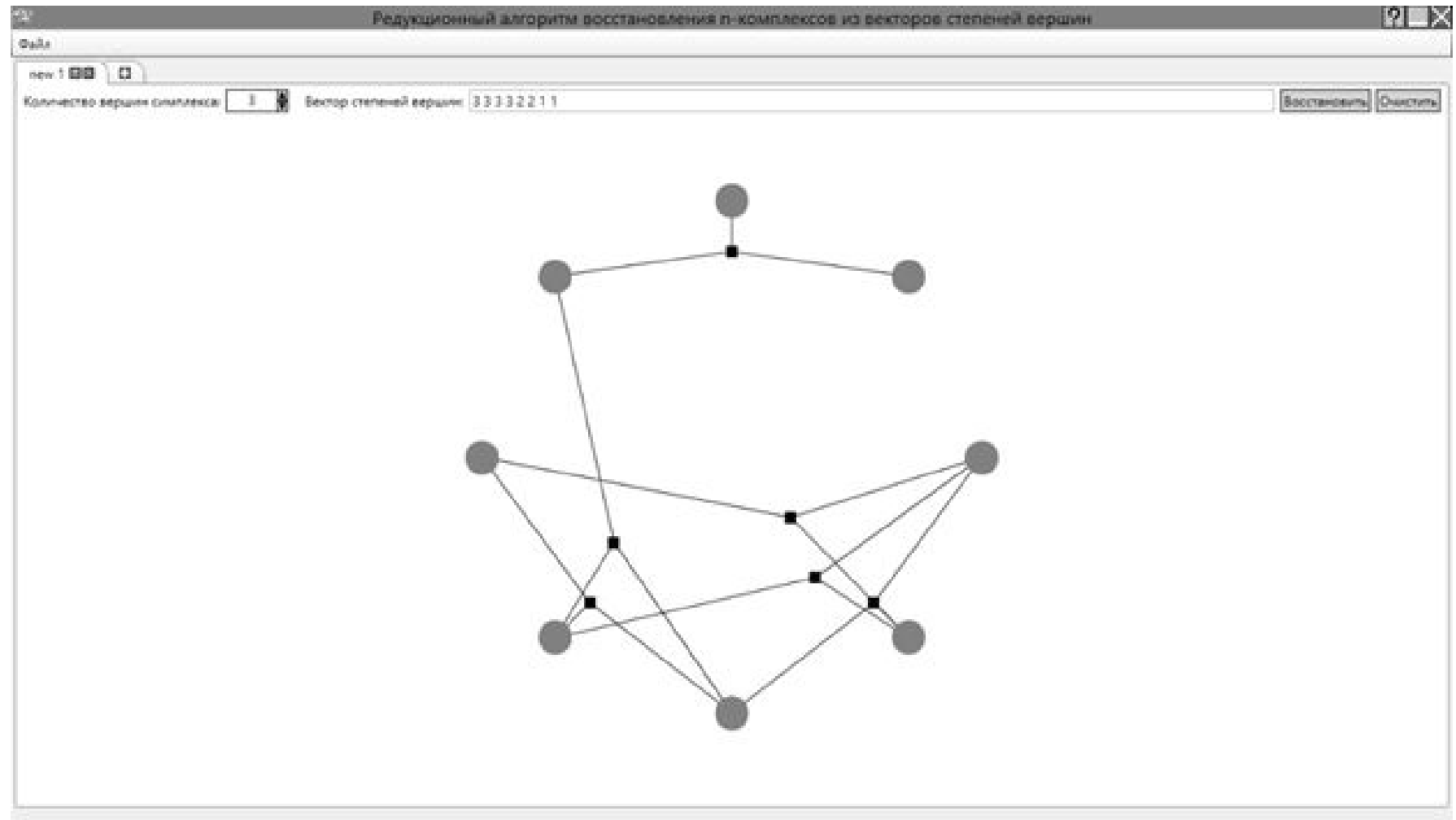

Рис. 1. Окно приложения после восстановления вектора в 3-однородный гиперграф

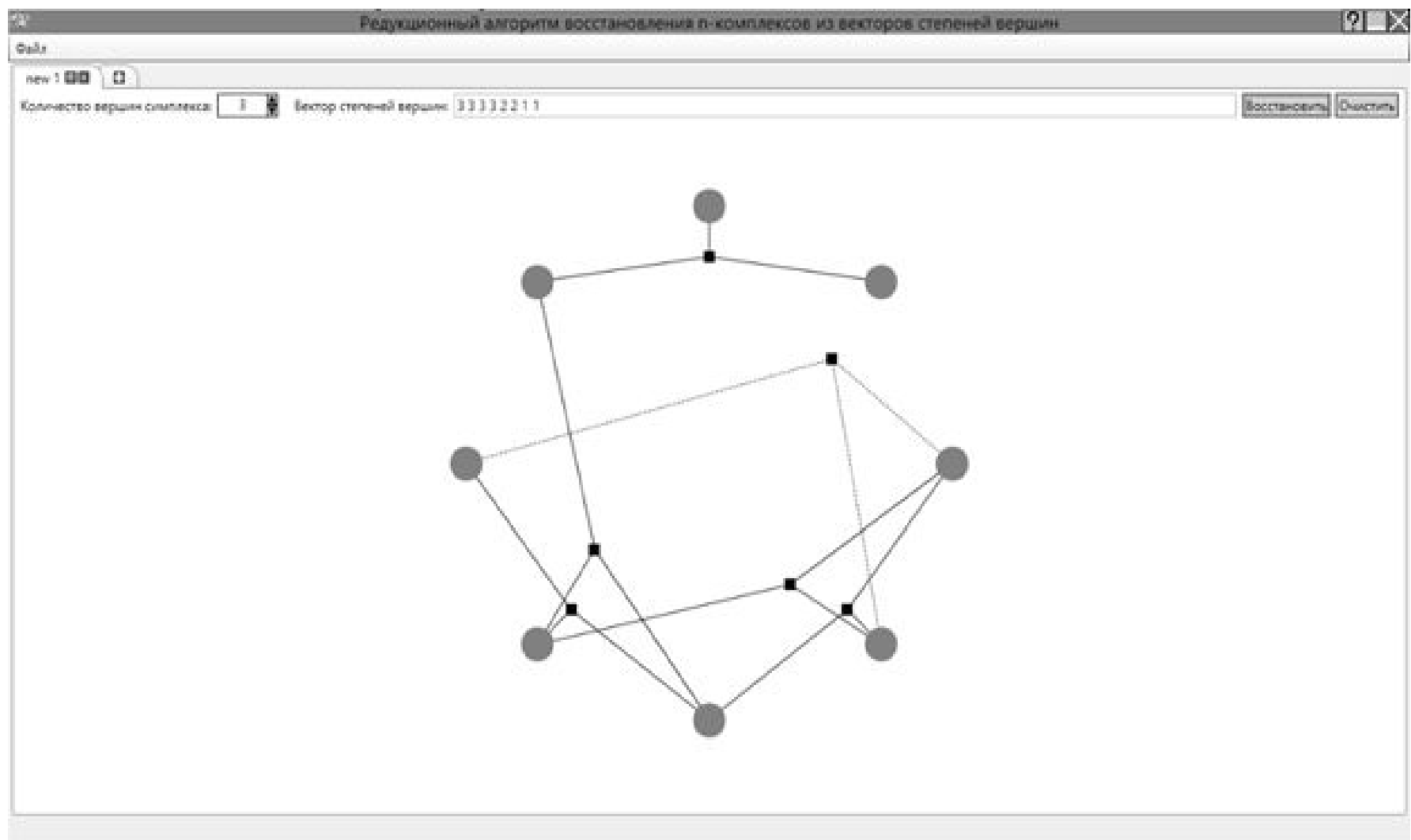

Рис. 2. Окно приложения при перетаскивании гиперрёбер 3-однородного гиперграфа 


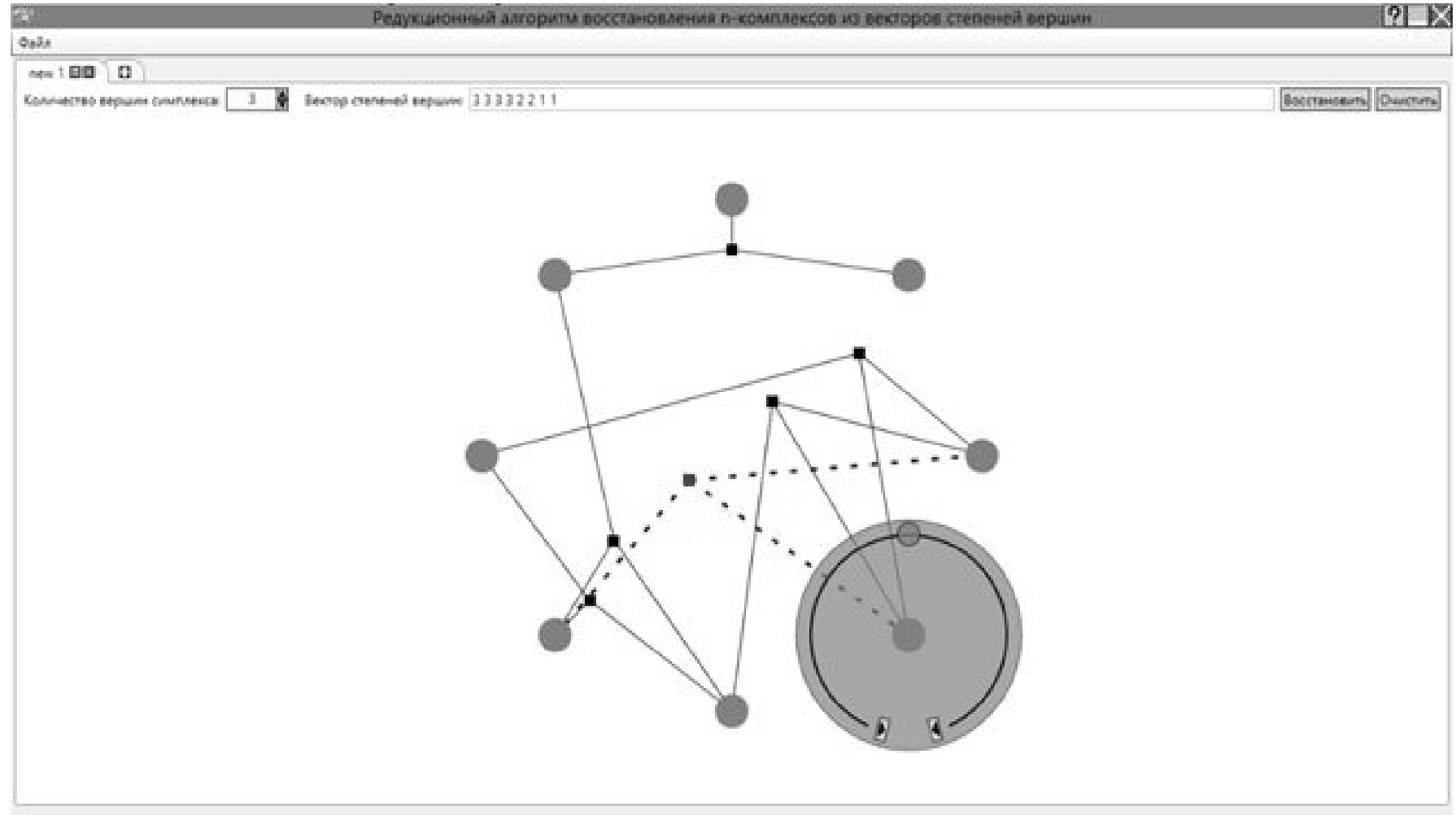

Рис. 3. Окно приложения после перетаскивании гиперрёбер 3-однородного гиперграфа

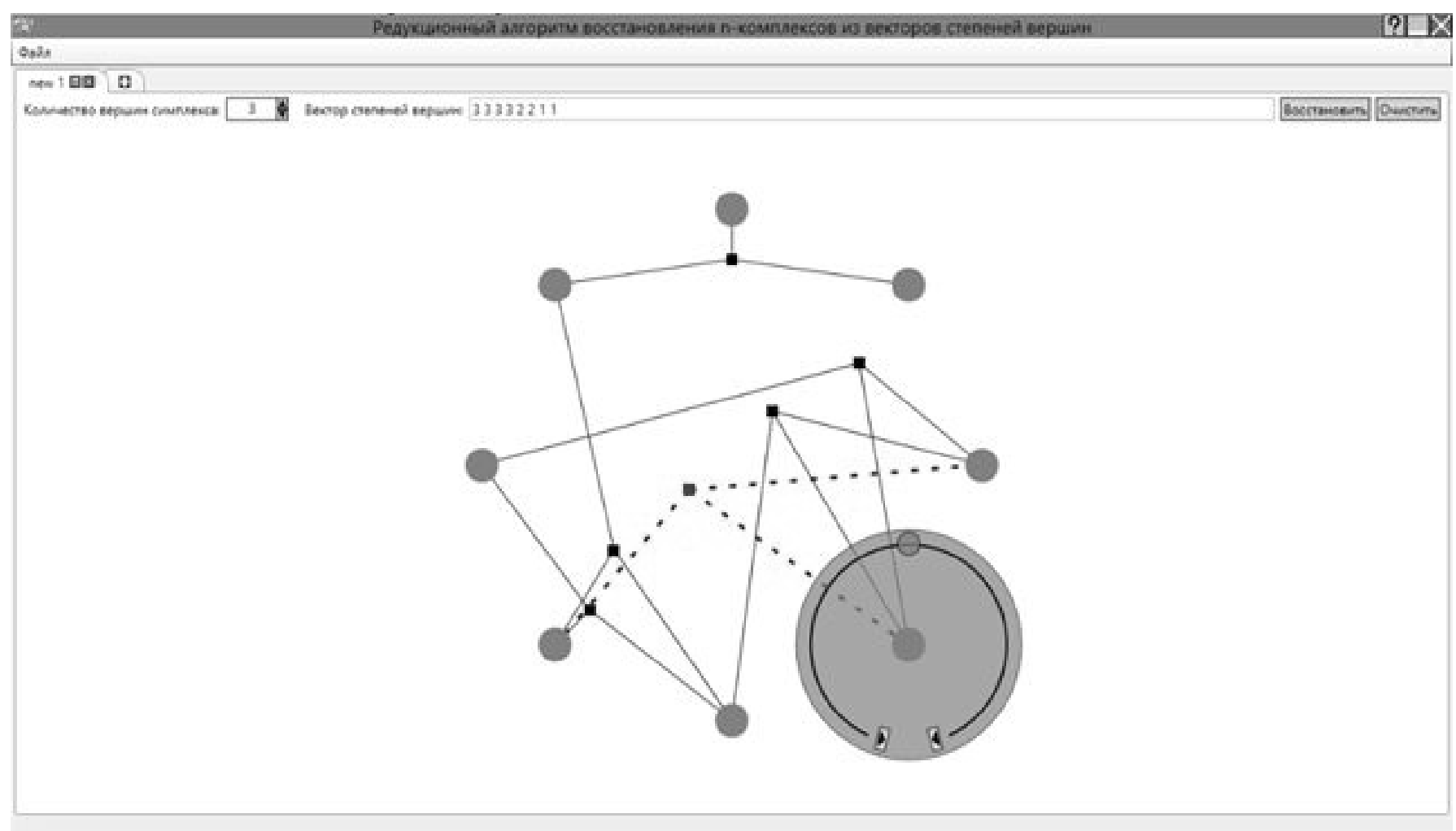

Рис. 4. Окно приложения при переключении инцидентных вершине гиперрёбер 
При запуске приложения появляется окно с элементом управления TabControl с одной вкладкой. В этой вкладке (как и в остальных, создаваемых пользователем) расположены элементы управления TextBox для ввода вектора степеней вершин, NumericUpDown (реализованный самостоятельно, так как WPF не предоставляет такой элемент управления) для указания количества вершин в одном симплексе, кнопка восстановления комплекса из введённого вектора и кнопка очистки восстановленного симплекса. Под вышеуказанными элементами управления располагается отображение восстановленного комплекса.

Пользователь может очистить вектор и восстановленный комплекс при помощи нажатия кнопки "Очистить". Также пользователь может работать с гиперрёбрами (перетаскивать их) при помощи зажатия мышью квадратика (центра симплекса) и перетаскивания квадратика по рабочей области вкладки.

Особое внимание уделено именно отображению полученных гиперграфов, для чего был разработан отдельный элемент управления.
У пользователя теперь существует возможность посмотреть гиперрёбра, которые инцидентны конкретной вершине. Для этого необходимо навести на вершину мышью, и спустя небольшое время появится элемент управления со скруглённым элементом управления наподобие ScrollBar и двумя кнопками, предназначенными для перехода на предыдущий или следующий симплекс. При нажатии кнопок или передвижении ползунка элемента ScrollBar, выбранный симплекс подкрашивается в синий цвет и меняет тип линии со сплошной на штриховую.

\section{Зак^ючение}

В дальнейшем планируется уточнить полученный редукционный алгоритм, для того чтобы он мог выступать в качестве необходимого и достаточного критерия реализуемости вектора в гиперграф.

Также интерес представляет и новый способ отображения гиперграфов, что предоставит дополнительные возможности исследователям в этой области.

\section{ЛИТЕРАТУРА}

1. Хакими С.П. 0 реализуемости множества целых чисел степенями вершин графа. М.: Мир, Кибернетика сб. нов. сер., вып. 2, 1966.

2. Миронов А.А. 0 реализуемости наборов чисел в граф и свойства графов с заданным набором степеней вершин // Тр. Гор. ГУ, 1981.

3. Миронов А.А., Мокряков А. В. Двумерные комплексы полностью описываемые степенями вершин // Труды Института системного анализа Российской академии наук. 2006. № 10. С. 178-188.

4. Mironov A.A., Mokryakov A. V., Sokolov A. A. About Realization of Integer Non-Negative Numbers Tuple Into 2-Dimensional Complexes // Applied and Computational Mathematics. 2007. T. 6. № 1. P. 58-68.

5. Mokryakov A.V., Tsurkov V. I. Reconstructing 2_Comlexes by a Nonnegative Integer_Valued Vector // Automation and Remote Control. 2011. V. 72 . No 12. P. 25412552.

6. Зыков А.А. 0 некоторых свойствах линейных комплексов // Мат. с6. 1949. Вып. 24 (2). С. 163-188.

7. Зыков А. А. Гиперграфы // УМН. 1974. Т. XXIX. № 6 (180). С. 89-154.

8. Kostyanoi D.S., Mokryakov A. V., Tsurkov V. I. Hypergraph Recovery Algorithms from a Given Vector of Vertex Degrees // Journal of Computer and Systems Sciences International. 2014. T. 53. № 4. P. 511-516.

9. Гурченков А.А., Костяной Д. С., Мокряков А. В. Редукционные методы восстановления некоторого класса гиперграфов // Инженерный журнал: наука и инновации. 2014. № 6 (30). С. 1.

10. Мокряков А.В., Селин П. С., Цурков В. И. Минимакс и восстановление по вектору в графах. М.: Физматлит, 2017. 309 с.

11. Egorova E.K., Mokryakov A. V., Vang L. Development of Hypergraph Theory // Journal of Computer and Systems Sciences International. 2018. V. 57. P. 109-114.

12. Mokryakov A. V. Hypergraphs as Algebraic Structures // Journal of Computer and Systems Sciences International. 2011. T. 50. № 5. P. 734-740.

13. Егорова Е.К., Есенков А. С., Мокряков А. В. Операции над к-однородными гиперграфами и их векторы степеней вершин // Известия РАН. Теория и системы управления. 2020. № 3. С.75-80.

14. Egorova E.K., Mokryakov A.V., Suvorova A. A. The Concept of Data Encryption Using Extreme Uniform Hypergraphs // Abstracts18th International Conference "Aviation and Cosmonautics - 2019". 2019. P. 409.

( Берецкий Игорь Сергеевич ( ISberetskij@mai.ru ), Ирбитский Илья Сергеевич ( ILSirbitskij@mai.ru ), Егорова Евгения Кирилловна ( egorovaek@mati.ru ), Мокряков Алексей Викторович (MokryakovAlVik@gmail.com ). Журнал «Современная наука: актуальные проблемы теории и практики» 